\title{
ENHANCED TELECOM OPERATION MANAGEMENT SCENARIOS FOR IMS NETWORKS
}

\author{
Errais Mohammed ${ }^{1,2}$, Raouyane Brahim ${ }^{1,2}$, Mostafa Bellafkih ${ }^{1}$ and Ramdani \\ Mohammed $^{2}$ \\ ${ }^{1}$ Institut national des postes et télécommunications (INPT), Rabat, Morocco \\ Mahammed_errais@yahoo.fr \\ ${ }^{2}$ Faculté des sciences et techniques de Mohammedia (FSTM), Mohammedia, Morocco \\ Raouyan_brahimeyahoo.fr
}

\begin{abstract}
The integration of IMS networks will enable telecom operators to evolve in a manner transparent to the incessant demand for the multimedia services. However the QoS management mechanisms defined for IMS networks are considered poor in oversight and monitoring real-time services. Moreover the eTOM Framework includes the scenarios of monitoring service delivery that enable real-time tracking services being supplies. These specifications are standard and contain no specification for IMS networks. We propose in this paper a new approach to monitoring of IMS networks, and eTOM process based, the monitoring architecture is deploying by the WSOA concept.
\end{abstract}

\section{KEYWORDS}

Enhanced Telecom Operation Management (eTOM), IP Multimedia Subsystem Network (IMS), Network Management, Quality of Service (QoS), Web Services (WS), Service Oriented Architecture (SOA).

\section{INTRODUCTION}

The current trend of telecom operators is the integration of the NGN solutions for the services deployment and networks management. In this context, the IMS [1] networks offer innovative solutions for multimedia services deployment regardless the type and topology of the network.

Indeed, the IMS networks include a new architecture for network management that defines a set of controls entities and service provision capable of maintaining a high level of access and security. However, the QoS management entities defined in the 3GPP specifications mainly focus on providing service without mechanism for real-time monitoring of services provided. So, the IMS is unable to identify and correct the deterioration to the QoS of current service.

Furthermore, the eTOM [2] Framework includes the monitoring scenarios of real-time services, based End-to-End processes. These scenarios are generics without specifications for IMS networks.

The eTOM Framework and the IMS networks combination enable operators to take advantage of the benefits of the IMS in service provisioning, and the ongoing supervision of services through eTOM processes. We propose in this paper a new approach for monitoring IMS networks eTOM

DOI : 10.5121/ijngn.2011.3202 
process based, and use the web services exposed via through SOAP [3] and WSOA [4] concept to reflect distributed architecture of eTOM layer.

In order to present all works of research and implementation done. In the first part of this paper we focus on QoS management mechanisms defined in the 3GPP specifications. Then we propose the processes defined in the eTOM framework in particular the scenarios dedicated to supervision and monitoring of services provided. Subsequently, we will present our new approach for monitoring before defining our system architecture implemented. Finally we will present the experiences and results.

\section{Service Provisioning in IMS Network}

The IMS system covers all the existing capabilities of voice, data and messaging, will also facilitate new media services such as video, with security, high quality and reliability according to the descriptions contractual SLA (Service Level Agreement) [5]. The IMS store many application servers (AS) in HSS (Home Subscriber Server) with their locations, to provide several servers.

The supply operation in IMS or service delivery is initiated by SIP signalling protocol [6], service requests are submitted using SDP [7] for negotiating all media parameters (Codec types, Communication ports, QoS type, and bandwidth). Afterward, the Diameter protocol [8] is using to reserve QoS requested by configuration of networks entities, and finally the media stream follow reserved path which meets all constraints.

As described (Figure 2), the offer of service begins with SIP INVITE sent by the client to GGSN gateway related over the NGN (Next Generation Network), which may be edge router in general case, after it through the chain IMS: P-CSCF and S-CSCF and AS (Application Server).

The QoS management is always presented before the media flow or specifically before sending the SIP message (200 OK); P-CSCF must be sure that resources are fully reserved, the PCRF (policy control and charging rules function) [9] entity must contacted the PCEF (Policy and Charging Enforcement Function) [10] resides in GGSN, which should install/ update all roles or policies.

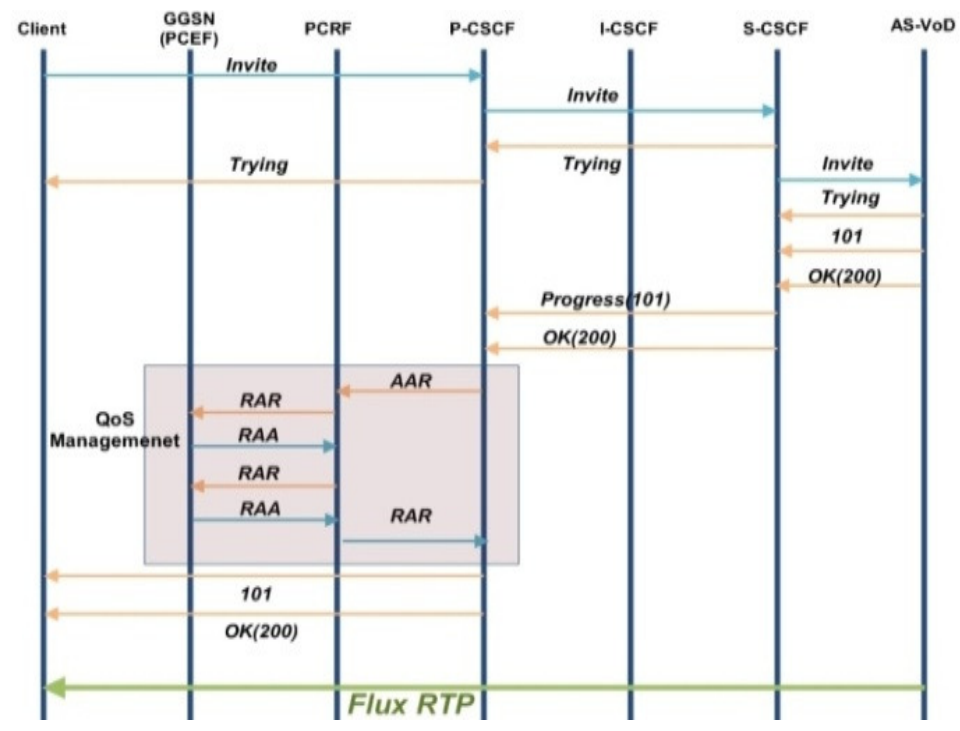

Figure 1. QoS Management in IMS Networks (VoD use case) 
Dialogue P-CSCF/PCRF based on Diameter for sharing requirements of the session, and between PCRF/PCEF for translating the information to a policy, that is the procedure followed for managing QoS in IMS architecture. Finally all managed resources are supporting new policies and configured with a QoS model for both paths upstream/downstream.

The management model as DiffServ [11] or IntServ [12] are chosen by the border routers or the GGSN, the PCRF is the only means QoS requirements, and the PCEF can choose the proper QoS management to implement this policy in the routers from the state and the model supported by the network and it infrastructure.

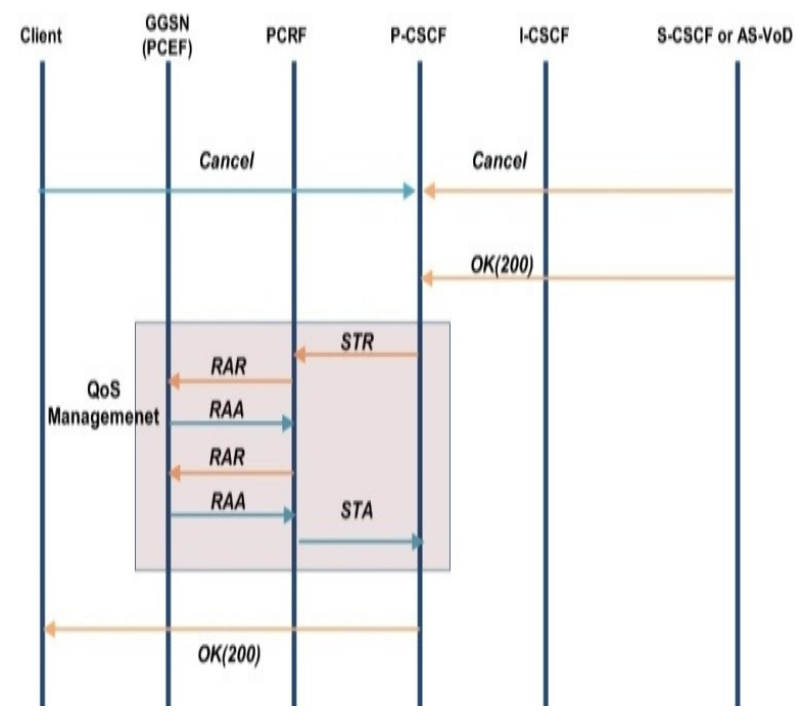

Figure 2. Diameter,SIP-based signaling for IMS sessions release

At the end of the session, the P-CSCF acquitted easily the stopping of service as well inform the GGSN to release resources for other applications, the Diameter message (STA) contains just identity of the session, that suspend resource reservation and QoS management model, finally the applied role for the session will be removed along the path followed by the media.

3GPP standards propose a QoS provisioning system without monitoring mechanism after delivery of services. Indeed, the 3GPP specifications focus on providing services such as resources reservation, but without worrying about the behavior-level of follows. The IMS as network control layer is unable to detect QoS deteriorates and resolve the problem. Also support neither class of services nor customer, without SLA definition and respect terms.

\section{ENHANCED TELECOM OPERATION MANAGEMENT (ETOM) FRAMEWORK}

\section{1. eTOM Framework presentation}

The eTOM Framework represent the whole of a service provider's enterprise, and positions this within its overall business context. The eTOM Architecture divides the relationship customer/operator in two main areas (Figure 3): horizontal processes used to manage customer contact or manage the supply chain; it defined the constitutional architecture as well as TMN (Telecommunication Management Network), layers (Costumer, Service, and Resource). And 
other vertical represent processes end-to-end groupings, which includes all operations management especially the 'Operations' party or FAB (Fulfillment, Assurance, Billing).

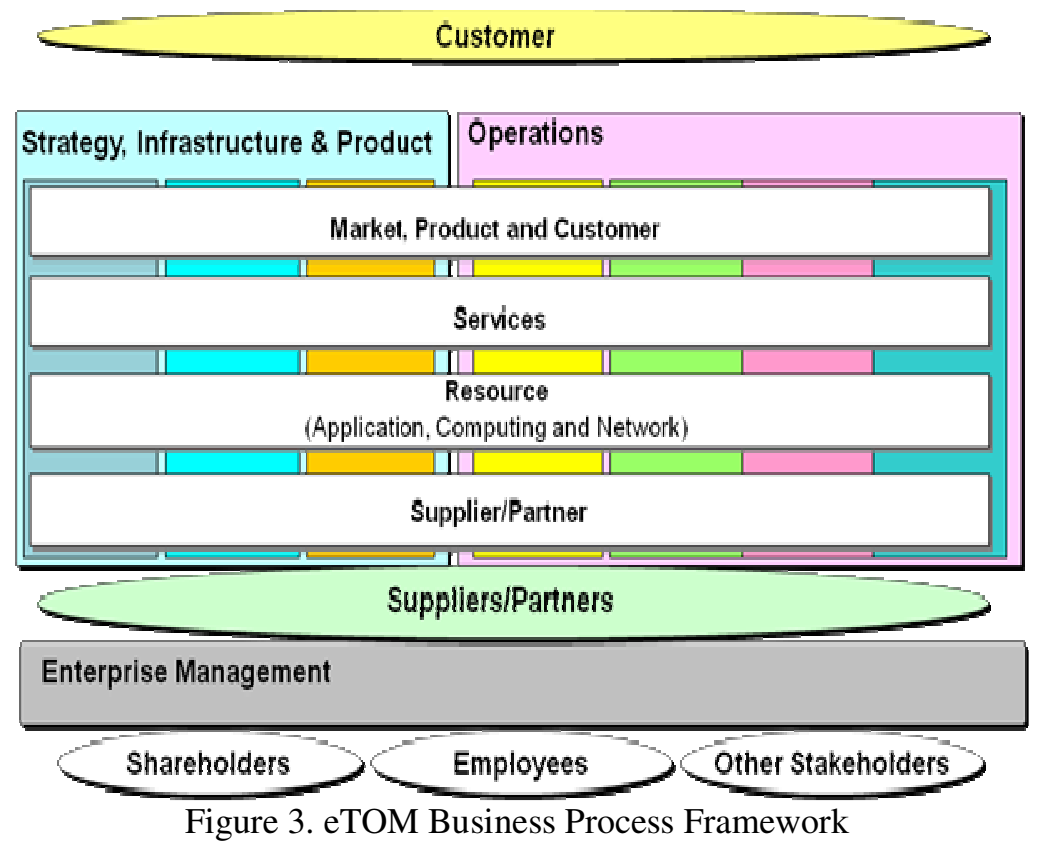

An operation between customer and enterprise (operator) will be translated in eTOM processes grouping, which operates the both layers to define the functional components, and End-to-End processes witch perform and fulfill the operation.

\subsection{Ordering process flow}

The eTOM develop and manage the supply chain as shown in ordering operation [13] (Figure 4), all implemented processes interact sequentially in the scenario and exchanging information between layers (Customer, Service, and Resource).

The Fulfillment process (ordering) is only one scenario of many possible ways of service delivery. The interface 'CRM' (Customer Relationship management) accepts the request and attempt to verify the customer credibility and ensure service availability, before sending the process to 'SC\&A' (Service Configuration \& Activation) which tries to determine the class of customer (Platinum, Gold, Bronze, Silver) and propose a delivery solution in terms of logical and physical resources. Finally the process 'RP' (Resource Provisioning) will reserve resources for a service and client specific and notify all supply chain as described below (Figure 4). 


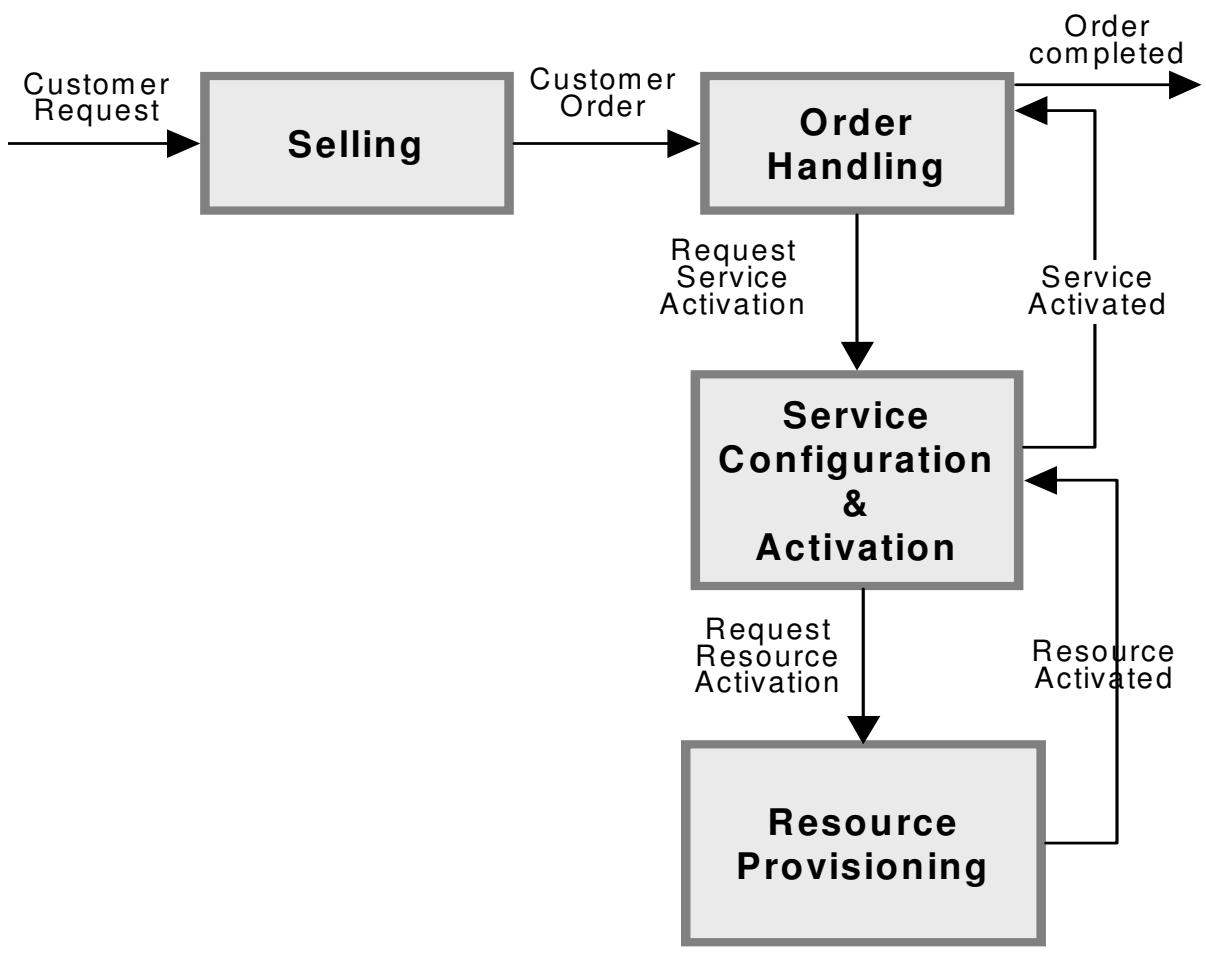

Figure 4.Ordering Processes as described by eTOM specification (Dynamic Ordering process)

In the fact, the IMS entities guarantee service delivery or fulfillment operation perfectly, the eTOM gives operators the ability to monitor the operation during and after for detecting any problems that appear (SLA violation, anomalies, security) by a main process vertical Assurance.

\subsection{SLA Verification process flow}

The scenario of SLA verification [5] as defined in the eTOM framework enable to identify the user satisfaction as well as the QoS provided in real time. And enable correct the deterioration in the shortest time.

The scenario of SLA verification requires a composition and cooperation of several processes (Figure 5) eTOM belonging to the interface "CSR". First, the process in resource layer collects key performance indicators (KPI), and applies a primary analysis then structure as need as the upper layer processes. Then depending on type of service, the appropriate quality indicators are defined and calculated before being transmitted to processes in Customer layer. Finally the customer profile is loaded to identify the thresholds defined in the SLA specific to service requested. These thresholds are applied to quality indicators calculated and the following results, the overall report of the verification. 




Figure 5. SLA Verification Processes as described by eTOM specification

\section{Projection eTOM FrameWork on IMS Platform}

\subsection{Work Description}

The projection of the eTOM framework in the IMS platform requires the integration of an effective methodology for a transparent communication between different entities of the eTOM and IMS. Indeed it is important to identify the scenarios to model, but also the necessary information and the technology using for implementing this approach.

The modelling approach is performed as follows:

- Business Process: The deployment eTOM process requires technological tools capable of supporting processes operations and facility communication by a bus. The tools allow absolute sharing between the three layers Resource, Customer and Service in accordance with the specifications of the TM Forum. So, the ideal solution is SOA (Service Oriented Architecture) [4] architecture, that enables deployment of EJB [14] modules which expose processes as Web Services and ensure communication by SOAP/XML.

- Orchestration: The orchestration between the web services exposed by the EJB modules is realized by a BPEL language [15]. The BPEL ensure the mapping and synchronization between web services related to operation.

- Data presentation: The information structuring and deployment is the most critical step in the modelling phase, by aggregate a set of data coming from multitude of components and technologies. So, different information models are necessary as follows:

- SID (Shared Information Data) [16]: for representing information model dedicated to eTOM processes, which reflect all data presented in each layer (Customer, Service, Resource), and their information from network entities. 
- Business information: for structuring the information related to customers as SLA or other profile data (CoS, QoS, Compte); all information will integrate as XMLDB.

- Log files: for all traces of performance indicators collected in each network entity or element.

- Key Quality Indicator: The choice of quality indicators is very important to enable the identification of user satisfaction. For Video service like VoD and IPTV, the two indicators MOS-A [18] and MOS-V [17] appear useful and reveal well customer satisfaction.

\subsection{Functional Architecture}

These eTOM processes (Figure 6) will be activated sequentially. In Assurance layer, the processes will interact for monitoring operation and use all network data information. In order to link the eTOM processes to the IMS network, a new components entitled "Configuration, \& Monitoring Component" and "Signalization Component" are required, which ensure a flexible communication between Business processes and network entities.

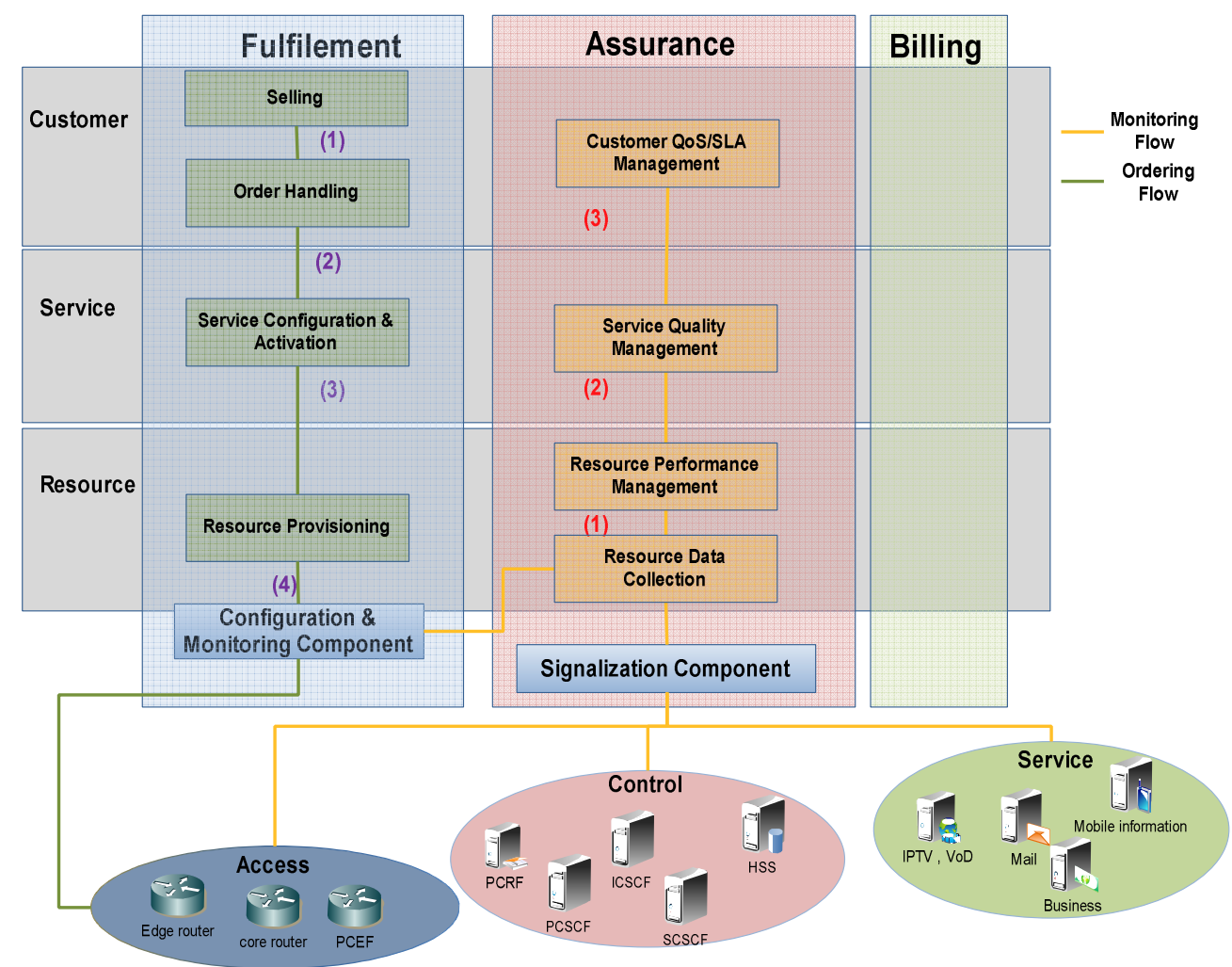

Figure 6.Functional Architecture

The diversity of entities and their various communication protocols require a set of components which can interpret all data form, and implement it for all monitoring operations. Although, the performance data collection and detection of service delivery should be executed in real time or near real. 
Three layered components are:

a) The components of interaction between the eTOM Framework and IMS network:

-Signalization Component: The eTOM processes must be linked with the various components of the IMS network, this component is responsible for detect the services launch and their type. This is performed via the S-CSCF as well as the Application Server. For identify the clients and their parameters (IP address, ports, Access technology, etc...).

-Monitoring \& Configuration Component: The component responsible for the configuration and activation of resources and identification of network performance based on parameters retrieved from signalization Component.

b) SLA Verification Component

- Resource Data Collection component (RDCP): This component is responsible for collecting performance indicators in resources. The Process contains several secondary processes according to level 4 in eTOM decomposition, which are responsible for gathering performance indicators and metrics for all service running in the network. After aggregation, the data performance must redistribute to other processes.

- Resource Performance Management component (RPM): The processes of component collect performance indicators; and provide a XML (Extensible Markup Language) reports featuring a structured view of the KQIs as well as threshold detection.

- Service Quality Management component (SQM): This component performs a mapping of performance indicators; it identifies for each service its quality indicators before determining appropriate operations to be performed to calculate KQIs. And used to estimate QoS and identify causes of failures in resources or missing capacity.

- Customer QoS/SLA Management component (CQSM): This component is responsible for the SLA verification. After retrieve the quality indicators from the Service Quality Management component and receiving the preliminary reports, it imports the client profile as well as SLA parameters to identify threshold levels for comparison purposes. It handles also report to the management server and provisions a comprehensive report on the service (Metric, KQIs, KPIs, Resource, etc. ...).

c) Ordering Components:

- Selling: The process is responsible for receiving client requests. It generate for each request received a structured report include the requirements of QoS and user profile.

- Order Handling: This process analyzes the client requests and decides according to his class and nature of the service the actions to execute.

- Service Configuration \& Activation (SCA): The process identifies possible solutions in terms of QoS depending on the service requested, the class of the client and resources state. 
- Resource Provisioning (RP): This process is responsible for resource configuration (physical and logical) based on the solution proposed by Service Configuration \& Activation process.

\section{SYSTEM ARCHITECTURE}

The implementation of approach requires adequate technological tools, which are able to maintain high performance, and allow a minimum cost in terms of time and resources.

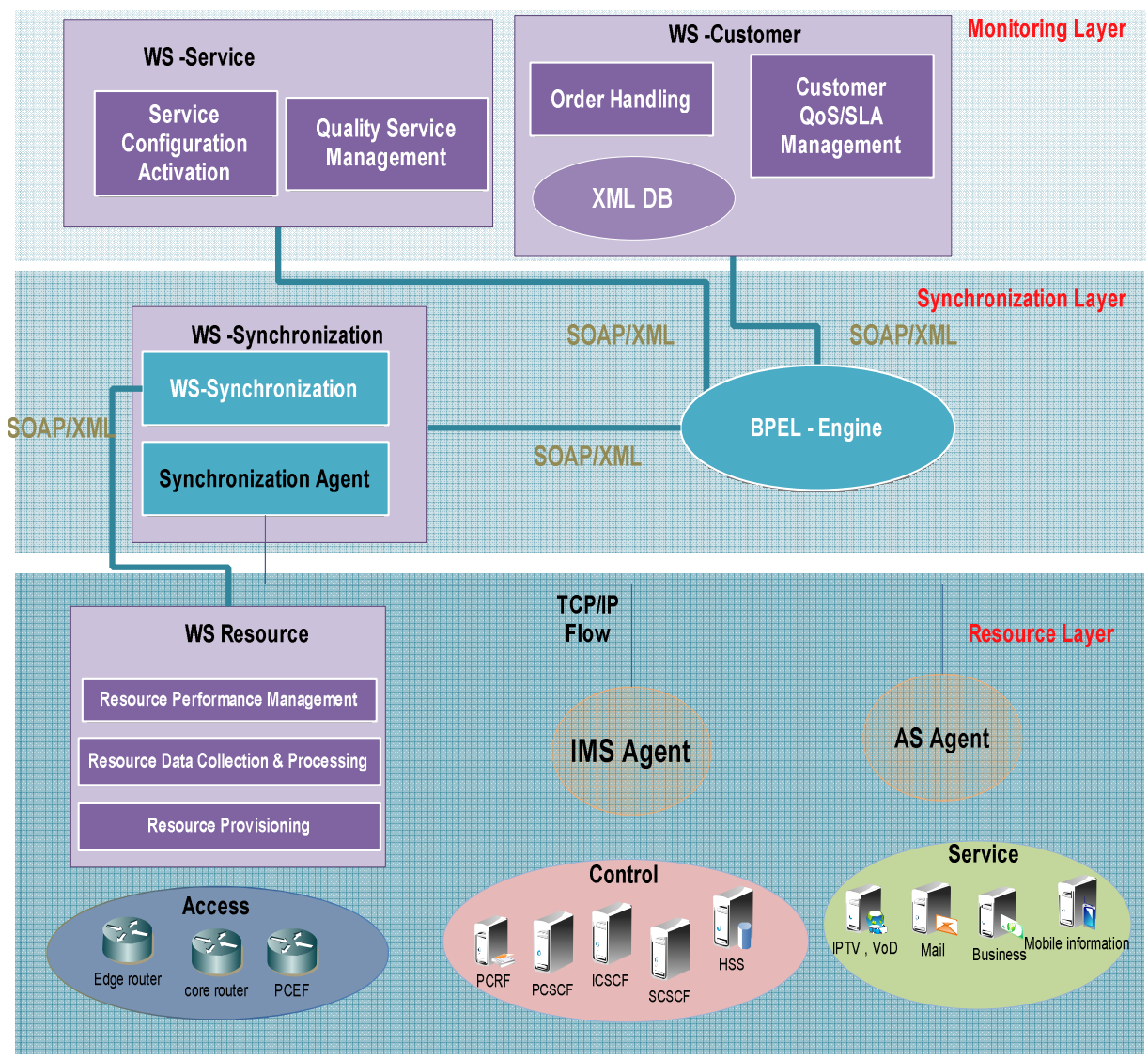

Figure 7. System Architecture

The distributed system architecture must equilibrate loads and reduce the time needed for communication between all components.

Indeed we define three functional levels (Figure) each one focuses on a particular aspect:

- Resource Layer: It focuses on the collection of key performance indicators as well as activation and configuration of resources. It brings together different modules dedicated to identifying the parameters of services provided by IMS entities and network status (WSResource, IMS agent, AS agent). 
- Synchronization Layer: this module allows synchronization between modules defined in the platform.

- Monitoring Layer: This layer is the top level of the architecture system that allows performance information collected to identify the state of service and the proposed solution for QoS.

The eTOM processes is translate into three EJB modules (Resource, Customer and Service). The eTOM processes are exposed as WS (Web Services) using SOA architecture. The orchestration between different web services is performed by a BPEL deployed in the synchronization layer. As well, the introduction of network agents is useful for represent the interaction between eTOM and the IMS (Figure 7).

\subsection{SOA Module}

- WS-Resource: This SOA module is composed of classes implementing operations defined in the eTOM Resource layer. It implements three main eTOM processes already discussed in the functional architecture: Resource Data Collection \& Processing, Resource Performance Management and Resource Provisioning. Both of them are exposed as web services. The resource module is deployed in network at routers level.

- WS-Service: This module implements the operations defined in the Service layer of eTOM. The module exposes the Customer QoS/SLA Management and Service Configuration $\&$ activation.

- WS-Customer: This module implements the functionality defined in the Customer layer of the eTOM. It exposes the Customer QoS/SLA Management, Order handling \& Selling Process which are related to business relation between operator and customer.

- WS-Synchronization: The module responsible for the synchronization of a side between IMS agent and Application Server agent, and another side between the resources modules deployed on routers. Indeed, this module exposes a Web service called "Synchronization" which includes reports of eTOM process of resource layer before transmitting to the BPEL-Engine.

- BPEL-Engine: The BPEL Engine module implements a BPEL process that invokes the web services described above and synchronize their interaction.

- Web interface : To monitor the SLA verification process, the BPEL-Engine features a web interface that allows to:

- Show messages exchanged between web services (XML/SOAP) and modules.

- List performance indicators collected from the network layer entity

- Monitor the activity and performance of physical resources such as network routers and logical entities such as CSCFs and the HSS (Home Subscribe Server).

- View the results of the audit and SLA verification, the customer class, and values of Quality indicators. 


\subsection{Monitoring Scenario}

The scenario of monitoring of the platform is as follows:

- The IMS agent detects the service request via SIP signalling exchanged between the control entities of the IMS. This last notifies the synchronization module which makes the recovery of service settings. Once the parameters of services and the client are identified, the synchronization module transmits a report to BPEL-Engine.

- The BPEL-Engine invokes the eTOM processes involved in the Ordering operation in the order specified in the functional architecture (Figure 7). The report of the operation is forwarded by the BPEL-Engine to synchronization module.

- The synchronization module transmitted the report of ordering to the resources modules to undertake the collection of key performance indicators. These indicators are recorded in real time in a log file identified by the session number. The Indicators are also analyzed based on the thresholds identified in the customer's SLA (report of ordering).

If the resource module identifies the end of session or critical values of the key indicators of performance, the operation of SLA verification is launched. The resource module transmitted the report to the synchronization module. This last includes the reports of all resources before transmitting them to the BPEL-Engine. The BPEL-Engine invokes the eTOM process involved in the SLA verification in the order specified in the functional architecture.

\section{EXPERIENCES AND RESULTS}

The platform has been validated by performing practical cases of multimedia services $(\mathrm{VoD})$ in an IMS network.

\subsection{Test band infrastructure}

The test bed is composed of:

- A core router and two edge routers (Linux boxes) defining a DiffServ-enabled network on which are connected an IMS terminal ad an Application Server;

- This network is controlled by the OpenIMS [18] system which is deployed in the core router Linux box;

- Management Server which includes the SOA modules Customer, Service and the web interface.

- Synchronization server that includes the Synchronization module and BPEL-Engine. 


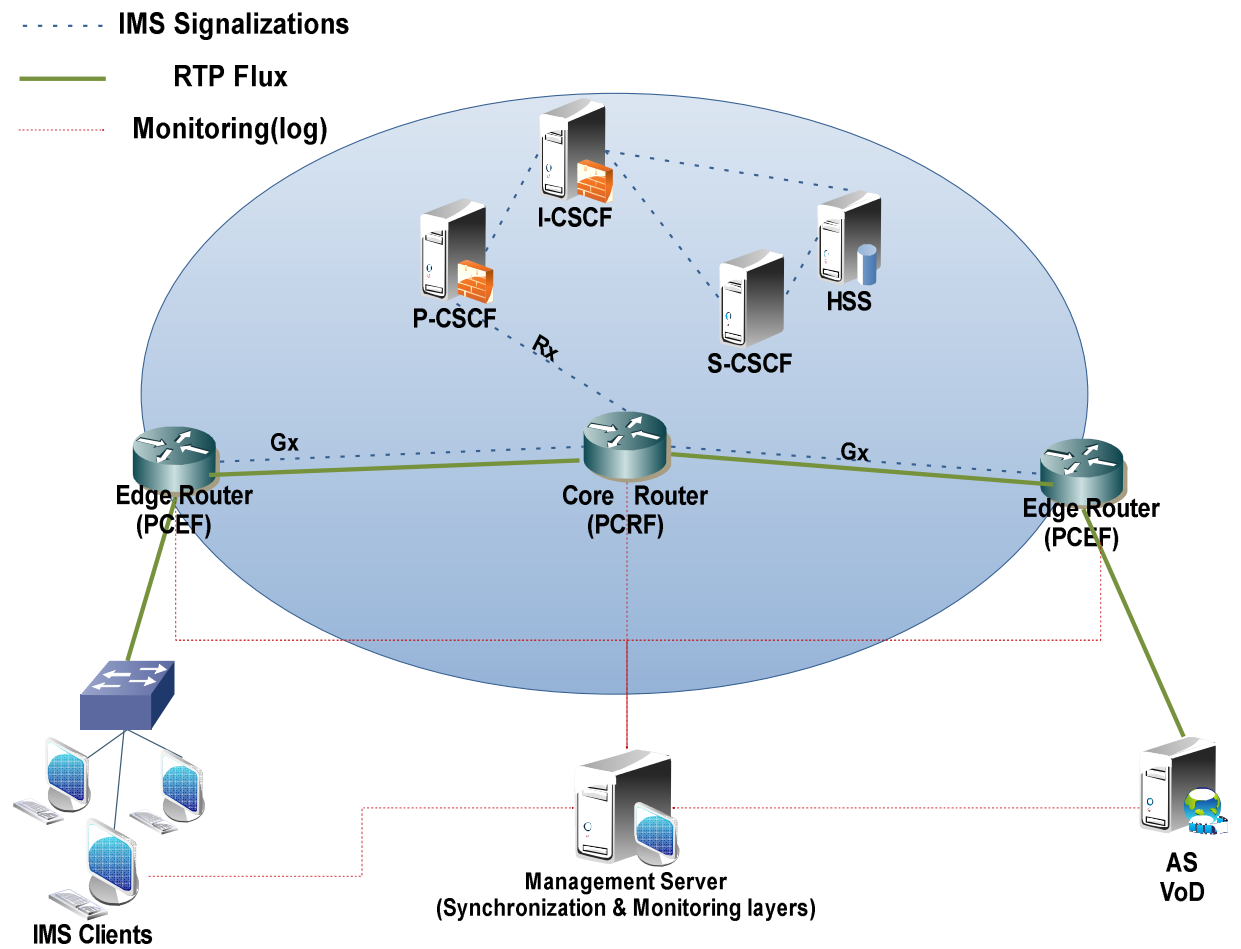

Figure 8.Test band Infrastructure

\subsection{Scenario}

Alice has registered in the IMS system with QoS classes Platinum. The goal is to perform SLA Assurance tests in two representative cases and to compare the results:

- $\quad$ No or only few competing services (FTP)

- Significant load of competing services

\subsection{Results}

Case 1: The QoS offered matches the SLA contract, perceived video quality is satisfying (Figure 9)

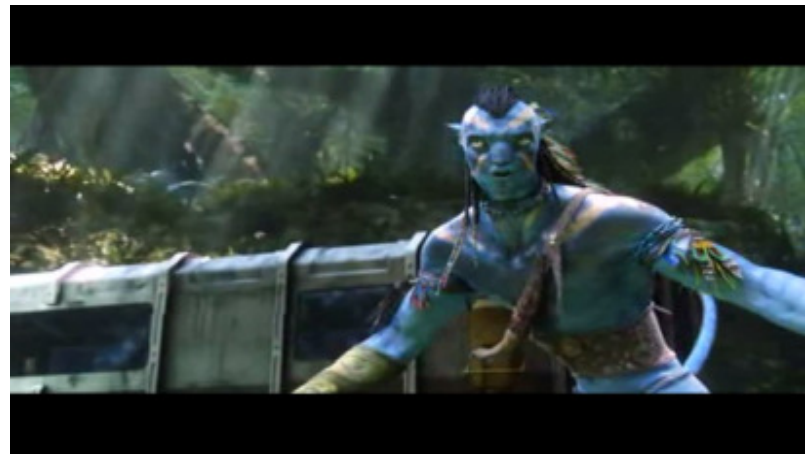

Figure 8.The image shows screen capture in case 1 
Case 2: competing services overload the routers: the queues fill in the gateways, impacting delay and jitter. Routers discard packets in excess; this causes static pixels in the video.

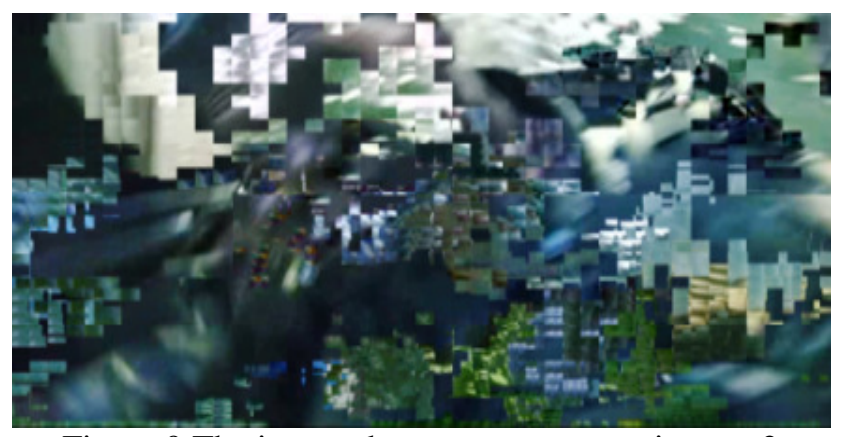

Figure 9.The image show screen capture in case 2

\subsection{Discussion}

The platform identifies the QoS successfully and customer satisfaction. However, it is necessary to evaluate the cost in terms of resource consumption and in execution time. We use routers are Linux machines (512MB in RAM and CPU $3.40 \mathrm{GHZ}$ ).

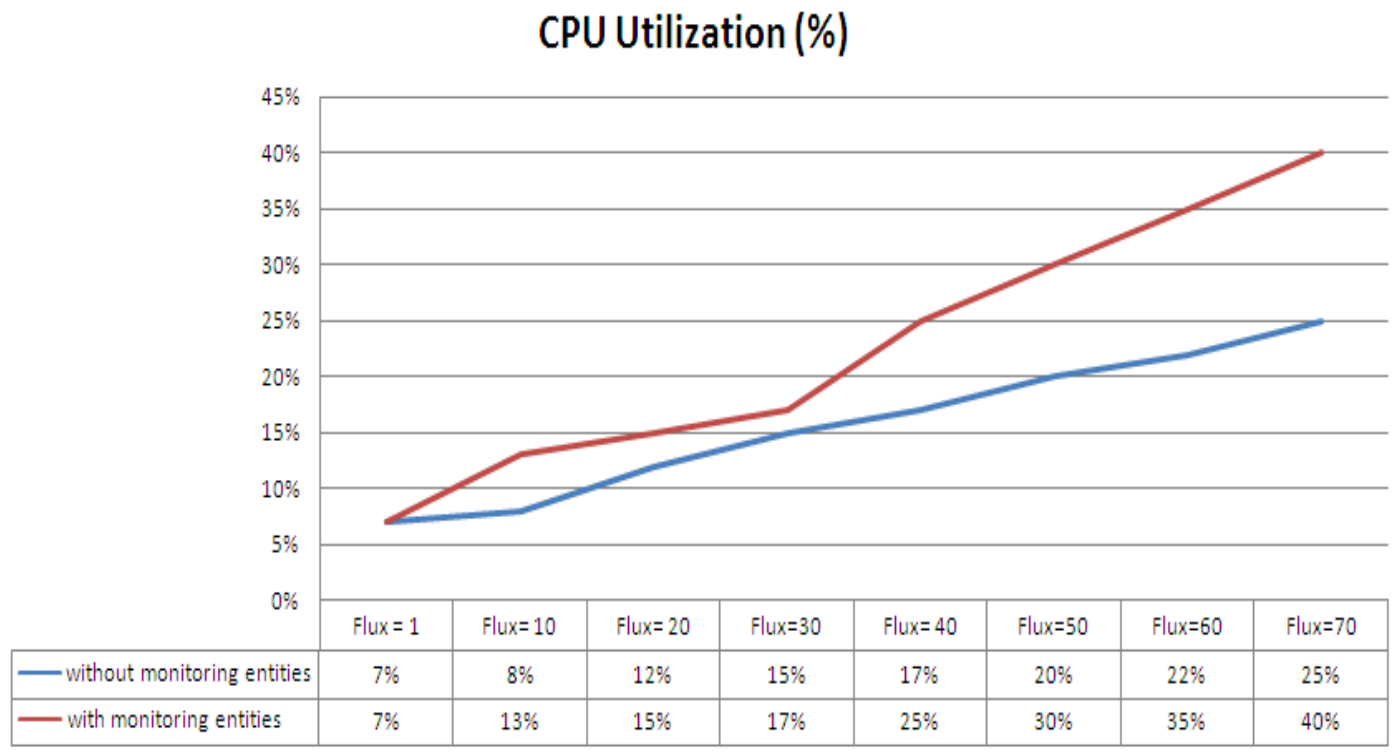

Figure 10.History of CPU utilization

Figure 10 describes the CPU utilization depending on the number of the flows in router. The activation of monitoring entities (Router Module) increases the consumption of CPU. However, the difference recorded in both cases (with and without monitoring entities) does not exceed a certain threshold (15\%). This explains the choice to minimize entities deployed directly on resources. 


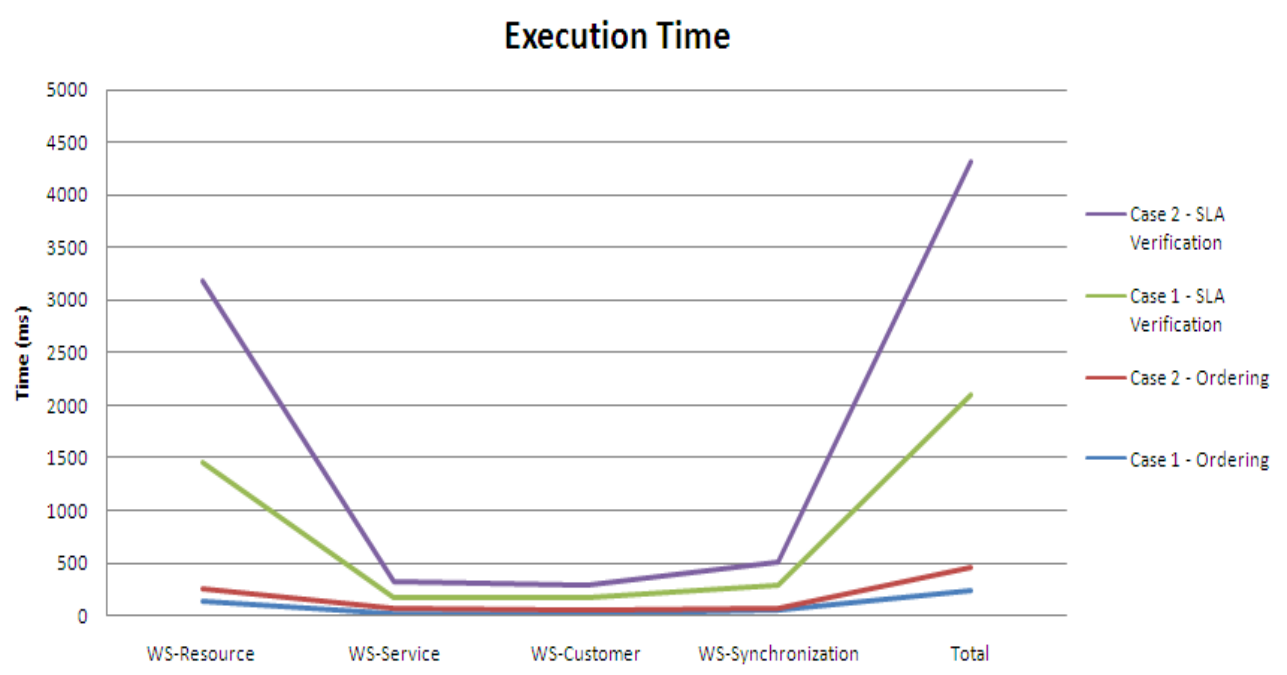

Fig. 11.The execution time of the platform in the various cases of experience

The execution time of the platform varies depending on the network state, and also operation type of eTOM. Indeed the time required for the ordering operation much less than the SLA verification; that, reflecting the complexity of the verification operation in particular the collection of performance indicators. Also the communication tools used through the components of the platform can ensure high reliability while minimizing the time required for the message exchange.

The cost in terms of execution time and resource required depends on the number of parallel service, but also the number of web services deployed via the SOA architecture. This explains our choice to opt for a distributed system architecture that offers a significant gain in terms of execution time. Also this architecture allows easy integration mechanisms to correct QoS deterioration in real time.

\section{Conclusions}

Our new approach complete 3GPP specifications for QoS management in the IMS. Indeed, the new approach allows continuous monitoring of services which takes into account several constraints as type of service, customer class and importance. Indeed, the choice of appropriate quality indicators for each service can accurately simulate a user satisfaction. Similarly, the SLA determines the thresholds to apply following type of customers what allows the operator to take appropriate decisions for each customer.

The SOA concepts implementation allows an absolute division between the different aspects of network management. Also our architecture deemed distributed aims from one side to reduce the system by supporting various media as required, but it also allows the integration a set of QoS correction processes for degradation detection. Indeed, the monitoring alone does not allow selfadministration of networks, it is therefore important to identify the troubles causes and QoS deterioration, before modelling and implementing appropriate processes for the automatic resolution or restoration. 


\section{ACKNOWLEDGEMENTS}

This work was supported in part by a grant from MÉDITELECOM.

\section{REFERENCES}

[1] Poikselka, M. and Georg M. (2009) The IMS: IP Multimedia Concepts and Services, John Wiley $\&$ Sons Inc. Chichester, England.

[2] ITU-T Recommendation M.3050.3 (2004) SERIES M: Telecommunications Management Network Enhanced Telecom Operations Map (eTOM) - Representative Process Flows (eTOM).

[3] Latest version of SOAP Version 1.2 specification: http://www.w3.org/TR/soap12, W3C Recommendation (Second Edition) 27 April 2007

[4] Mark Hansen, D. (2007) SOA Using Java Web Services, Prentice Hall.

[5] Enhanced Telecom Operations Map (eTOM) The Business Process Framework for the Information and Communications Services Industry, Addendum D: Process Decompositions and Descriptions Release 6.0 GB921 D; TMF.

[6] SIP: Session Initiation Protocol, June 2002, RFC 3261

[7] SDP: Session Description Protocol, April 1998, RFC 2327

[8] Korhonen, J., Tschofenig, H., Arumaithurai, M. Jones, M., Ed., and A. Lior, "Traffic Classification and Quality of Service (QoS) Attributes for Diameter",RFC 5777, February $2010 .$.

[9] 3GPP TS 29.210 V6.7.0 “Charging rule provisioning over Gx interface (Release 6)". 2006-12.

[10] 3rd Generation Partnership Project; Evolution of policy control and charging (Release 7), 3GPP TR 23.803 V7.0.0 (2005-09).

[11] An Architecture for Differentiated Services, RFC 2475

[12] The Use of RSVP with Integrated Services, RFC 2210

[13] SERIES M: Telecommunications management network Enhanced Telecom Operations Map (eTOM) -Representative process flows, ITU-T Recommendation M.3050.

[14] EJB 3.0 Specification: http://openejb.apache.org/3.0/ejb-30-specification.html.

[15] Business Process Execution Language Version 2.0.Public Review Draft, 23th August, 2006.http://docs.oasis-open.org/wsbpel/2.0/

[16] Shared Information/Data (SID) Model System View Concepts and Principles, GB926, Version 1.0, Release 4.0 January 2004.

[17] Bellafkih, M.; Raouyane, B.; Errais, M.; Ramdani, M.; , "MOS evaluation for VoD service in an IMS network," I/V Communications and Mobile Network (ISVC), 2010 5th International Symposium on, vol., no., pp.1-4, Sept. 30 2010-Oct. 22010.

[18] OpenIMScore - Open source implementation of IMS Call Session Control Functions and Home Subscriber Service (HSS) -http://www.openimscore.org/ 


\section{Authors}

Errais Mohamed received his Master from The Faculty of Sciences, Mohammed V University Agdal, Rabat, Morocco, in 2009. He is currently doing his $\mathrm{PhD}$ at FSTM, Mohammedia, Morocco, under the supervision of Prof. Mostafa Bellafkih. His research interests include network management and software development.

Raouyane Brahim is a $\mathrm{PhD}$ student at the Faculty of Science and Technology Mohammedia specializing in QoS and Network Management in IMS and NGN. His field of expertise is in computer software and hardware, including network, and performance computing.

Bellafkih Mostafa had his $\mathrm{PhD}$ thesis in computer science from the University of Paris 6 , France, in June 1994 and Doctorat Es Science in Computer Science (option networks) from the University of Mohammed V in Rabat, Morocco, in May 2001. His research interests include network management, knowledge management, AI, data mining and database.

Ramdani Mohammed had his $\mathrm{PhD}$ thesis in Computer Science from the University of Paris 6, France, in February 1994 and Habilitation in Computer Science from the University of Paris 6, France, in June 2001. His research interests include the, knowledge management, AI, data mining and database. 